\title{
Network based performance of GPS-only and combined GPS/GLONASS positioning under different sky view conditions
}

\author{
Salih Alcay $^{1} \cdot$ Cemal Ozer Yigit $^{2}$
}

Received: 2 October 2015/Accepted: 25 April 2016/Published online: 3 June 2016

(C) Akadémiai Kiadó 2016

\begin{abstract}
This paper offers a comprehensive assessment of network-based positioning results using GPS-only and combined GPS/GLONASS data under open to limited sky view conditions. Data analysis is performed in a short $(4 \mathrm{~h})$ and a long $(24 \mathrm{~h})$ term spanning observations from five GNSS stations using $10^{\circ}, 20^{\circ}, 30^{\circ}$ and $40^{\circ}$ elevation cut-off angles, which simulate the availability of satellites in different sky view conditions. Evaluation of the results reveals insignificant differences between the accuracy of the GPS-only and combined GPS/GLONASS solutions for daily observation in all sky view conditions. However the clear advantage of integrating GPS and GLONASS observation in a GNSS differential solution is apparent for short $(4 \mathrm{~h})$ observation periods, particularly at the highest elevation cut-off angle $\left(40^{\circ}\right)$.
\end{abstract}

Keywords GPS · GLONASS · Network-based positioning · GNSS network · Elevation cut-off angle

\section{Introduction}

In recent years the GLONASS revitalization has progressed steadily (2001-2011) and constellation of 24 satellites is completely deployed. This brings significant benefits to geodetic applications since it is possible to use the GLONASS system independently for positioning and on the other hand it gives opportunity to verify the GPS based solutions.

Salih Alcay

salcay@konya.edu.tr

1 Department of Geomatics Engineering, Engineering and Architecture Faculty, Necmettin Erbakan University, 42090 Konya, Turkey

2 Department of Geomatics Engineering, Gebze Technical University, 41400 Gebze, Kocaeli, Turkey 
In recent years great attention is placed by researches dealt with the contribution of GLONASS to the global positioning in both precise point positioning (PPP) (Choy et al. 2013; Dawidowicz and Krzan 2014; Yigit et al. 2014; Alkan et al. 2015) and networkbased positioning mode (Martin and Ladd 1997; Raffaela and Marco 2000; Bruyninx 2007; Wang and Wang 2007; Al-Shaery et al. 2011; Alcay et al. 2012; Zheng et al. 2012).

Bruyninx (2007), attempts to compare the GPS and GPS/GLONASS data analysis in regional network with operational 29 GPS and 13 GLONASS satellites. Results indicate that adding the GLONASS data to the GPS data does not significantly change the repeatabilities of any of the station coordinates. In addition using IGS and CODE orbits, similar results are obtained. Among the most recent ones, Alcay et al. (2012) analyzes the GLONASS-only, GPS-only and GPS + GLONASS positioning in various length of baselines. Analysis reveals that repeatabilities of all baselines by using GLONASS observations, corresponding to 20 operational GLONASS satellites, are not consistent when compared to the GPS and GPS/GLONASS. Nevertheless, Zheng et al. (2012) compare full constellation GLONASS-only and GPS-only results in two different networks. According to the results, GLONASS coordinates accuracies are slightly worse than that of GPS, reaching maximum $1.5 \mathrm{~mm}$ for the vertical (up) components. On the other hand, Choy et al. (2013) examines the benefits and challenges of a combined GPS and GLONASS precise point positioning (PPP) system in post-processed static and kinematic modes. The results confirm that for kinematic PPP the addition of the GLONASS observation improves the accuracy and precision of the position coordinates, whereas for daily static PPP, the benefits of integrating GLONASS are marginal. Yigit et al. (2014) offers a comprehensive statistical assessment of static PPP using GPS-only, GLONASS-only and combined GPS/GLONASS data. Evaluation of the results reveals insignificant differences in the accuracy and repeatability among the three satellite constellation solutions for 8 and $24 \mathrm{~h}$ observation times, whereas the superiority of the combined GPS/GLONASS solution is apparent for 1 and $2 \mathrm{~h}$ observation periods.

The accuracy and reliability of GPS-based positioning depends on, among others, the number of satellites being tracked and positioning dilution of precision (PDOP) values based on satellite geometry. Thus, positioning accuracy, reliability and coordinate repeatability is degraded in areas of low satellite visibility (e.g. urban canyons, deep open pit mine, and forestry). The addition of GLONASS, Galileo or BeiDou constellation will increase the number of visible satellites and can improve PDOP value. Therefore, to meet the need of high precision surveying applications, such as crustal deformation monitoring, monitoring of long-term behavior of engineering structures, a combined GNSS constellation (GPS/GLONASS, GPS/Galileo, GPS/BeiDou) is one of the aiding solutions. In this paper, we will evaluate the performance of GPS-only and combined GPS/GLONASS solutions at different elevation cut-off angles $\left(10^{\circ}, 20^{\circ}, 30^{\circ}\right.$ and $\left.40^{\circ}\right)$, which mimic the environments such as urban canyons or open-pit mines areas, for network-based positioning.

The paper is organized as follows: Following the introduction, the next section provides brief overview of the data processing, followed by an outline of the processing step of the utilized software. Then a detailed presentation of the results obtained from two observation datasets associated with various sky view condition scenarios. Finally, the last section summarizes key conclusions. 


\section{Data and processing method}

A network consists of five GNSS stations were selected for the study area. As shown in Fig. 1 GNSS stations are located in mid-latitude region. In addition, the stations, corresponding geographic coordinates and details of GNSS equipment's are provided in Table 1.

The GPS-only and combined GPS/GLONASS data processing were carried out using the Bernese GNSS Software (Version 5.2), which allows to process GPS-only, GLONASSonly and GPS + GLONASS observations. In this study, processes and parameters used in the programs were chosen according to Dach and Fridez (2016). The processing scheme displayed in Fig. 2 indicates the procedure implemented in this study.

While IGS final orbits were used for GPS-only processes, IGS and IGL final orbits were merged to be used for combined GPS/GLONASS based applications. Recently, accuracy of the GLONASS final orbits has improved and approached to GPS final orbits. Currently the accuracy of IGS final orbits is $2.5 \mathrm{~cm}$ for GPS and $3 \mathrm{~cm}$ for GLONASS (URL-1).

After the preparation of the required data, the first step starts with the processing of the pseudo-range observations for the receiver clock synchronization. If GPS and GLONASS data are processed simultaneously and the satellite clock file contains clock corrections also for the GLONASS satellites, the difference between GPS and GLONASS system time as an additional parameter for each station is estimated.

The main task after code processing is to form single difference observation files by selecting a set of baselines between stations using several strategies. The strategy applied here is based on the defined option to form 8 baselines (GOPE-LEIJ [ $\sim 218 \mathrm{~km}$ ], GOPEWROC [ $\sim 209 \mathrm{~km}$ ], GOPE-LINZ [ 182 km], GOPE-CAKO [ $410 \mathrm{~km}$ ], LEIJ-WROC [ $\sim 328 \mathrm{~km}]$, LEIJ-LINZ [ $\sim 365 \mathrm{~km}]$, CAKO-WROC [ $\sim 527 \mathrm{~km}]$ and CAKO-LINZ $[\sim 268 \mathrm{~km}])$. The second main process is to perform ambiguity resolution using Quasi-

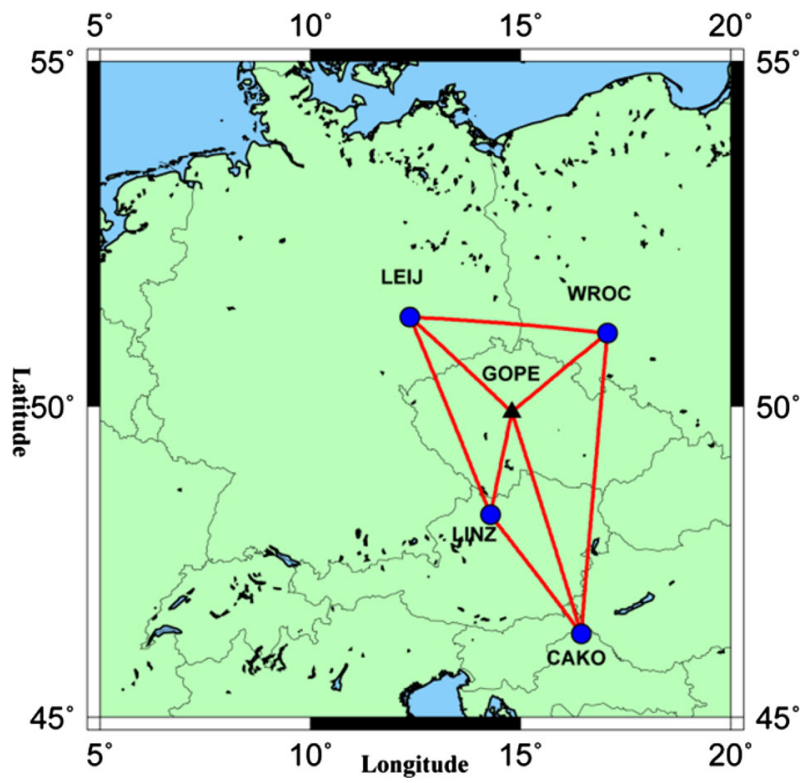

Fig. 1 Geographical location of the GNSS network 
Table 1 Details of GNSS receivers of the network sites

\begin{tabular}{llllll}
\hline Stations & $\mathrm{N}(\mathrm{Deg})$ & $\mathrm{E}(\mathrm{Deg})$ & $\mathrm{U}(\mathrm{m})$ & Receiver & Antenna \\
\hline GOPE (Ref.) & $49^{\circ} .91$ & $14^{\circ} .79$ & 592.6 & TPS NETG3 & TPSCR.G3 \\
CAKO & $46^{\circ} .39$ & $16^{\circ} .44$ & 222.1 & TRIMBLE NETR5 & TRM55971.00 \\
LEIJ & $51^{\circ} .35$ & $12^{\circ} .37$ & 178.4 & JAVAD TRE_G3TH & LEIAR25.R3 \\
LINZ & $48^{\circ} .31$ & $14^{\circ} .28$ & 335.0 & TRIMBLE NETR9 & TRM59900.00 \\
WROC & $51^{\circ} .11$ & $17^{\circ} .06$ & 180.8 & LEICA GR25 & LEIAR25.R4 \\
\hline
\end{tabular}

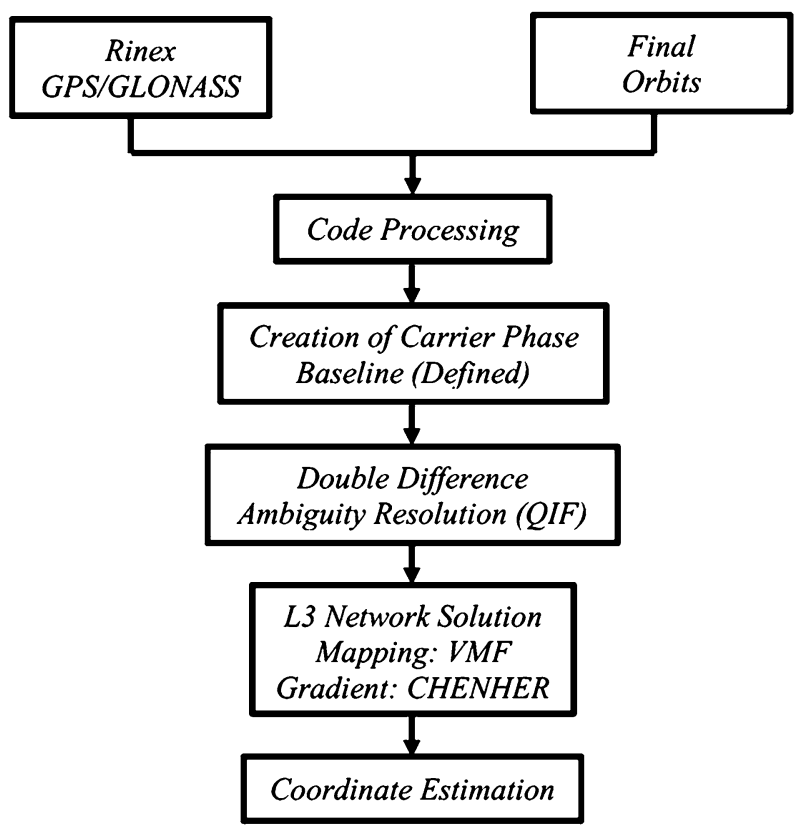

Fig. 2 Processing flow used in this study

Ionosphere-Free (QIF) method. In case of ambiguity resolution including GLONASS, only one ambiguity per iteration can be resolved. In addition in case of GLONASS, only ambiguities with the same channel number are resolved in version 5.2 (Dach et al. 2015). The main last step is the least-squares adjustment based on ionosphere-free linear combination (L3) network solution and coordinate estimations. In case of this step, Vienna mapping function (VMF) was used and the grid files for the coefficients of the VMF were downloaded from the website: http://ggosatm.hg.tuwien.ac.at/DELAY/GRID/VMFG/. In addition gradient estimation model applied here was CHENHER. Coordinates of four stations (CAKO, LEIJ, LINZ, WROC) were estimated based on GOPE station. In this study, once again, processes and parameters used in the programs were chosen according to Dach and Fridez (2016). More optimum results may be achieved using different parameters and different options. Since the focus of this study is to compare the performance of single 
system and a combined system, same processing strategy for all data sets was implemented.

\section{Results and analysis}

In this section following the scheme displayed in Fig. 2, the testing procedure and detailed results of two case studies undertaken to evaluate the performance of GPS-only and combined GPS/GLONASS solutions dealt with different sky view conditions are presented and discussed. Four tests were carried out for both long and short observation session applications, using different elevation cut-off angels to simulate four different sky view conditions. Firstly the elevation cut-off angle was set to $10^{\circ}$, representing the case of open sky view where no obstructions exist in the vicinity of the receiver, thus having maximum satellite availability. Secondly, semi-open cut-off elevation angle was set to $20^{\circ}$. Thirdly, limited sky view condition $\left(30^{\circ}\right)$ and finally an extremely limited sky view condition were assumed with high elevation cut of angle $\left(40^{\circ}\right)$, simulating an urban canyon environment, open-pit mine and forest area. In addition, we compare and discuss the accuracy of positioning and height estimation generated using the two constellation configurations for each elevation cut-off angle scenario.

\subsection{RMSE calculation procedure}

To aid interpretation, estimated station coordinates are transformed from geodetic cartesian $(X, Y, Z)$ coordinates to their corresponding topocentric $(e, n, u)$ equivalents. Analyses of the results in local geodetic frame can be physically meaningful due to the separation of the position and height. The common equations for the transformation are given as follows (Leick 1994)

$$
\left[\begin{array}{c}
\Delta n_{i} \\
\Delta e_{i} \\
\Delta u_{i}
\end{array}\right]=R\left(\phi_{0}, \lambda_{0}\right)\left[\begin{array}{c}
\Delta X_{i} \\
\Delta Y_{i} \\
\Delta Z_{i}
\end{array}\right],\left[\begin{array}{c}
\Delta X_{i} \\
\Delta Y_{i} \\
\Delta Z_{i}
\end{array}\right]=\left[\begin{array}{c}
X_{i}-X_{0} \\
Y_{i}-Y_{0} \\
Z_{i}-Z_{0}
\end{array}\right] .
$$

$X_{0}, Y_{0}, Z_{0}$ are the geocentric coordinates of the topocentric origin, which is derived from the mean of 30 daily solutions using all GPS and GPS/GLONASS datasets based on elevation cut-off angle of $10^{\circ}$, and $X_{i}, Y_{i}, Z_{i}$ refer to the geocentric coordinates obtained from each case solution. In Eq. $1, R\left(\varphi_{0}, \lambda_{0}\right)$ is the rotation matrix composed as follows

$$
R\left(\varphi_{0}, \lambda_{0}\right)=\left[\begin{array}{ccc}
-\sin \varphi_{0} \cos \lambda_{0} & -\sin \varphi_{0} \sin \lambda_{0} & \cos \varphi_{0} \\
-\sin \lambda_{0} & \cos \lambda_{0} & 0 \\
\cos \varphi_{0} \cos \lambda_{0} & \cos \varphi_{0} \sin \lambda_{0} & \sin \varphi_{0}
\end{array}\right]
$$

where $\varphi_{0}$ and $\lambda_{0}$ are geodetic latitude and longitude of the topocentric origin, respectively, which is calculated from their geocentric cartesian coordinates $\left(X_{0}, Y_{0}, Z_{0}\right)$.

Root mean square error (RMSE) for the north, east and up components are:

$$
\operatorname{RMSE}_{n}=\sqrt{\frac{\sum_{i=1}^{m} \Delta n_{i}^{2}}{m}}
$$




$$
\begin{aligned}
& R M S E_{e}=\sqrt{\frac{\sum_{i=1}^{m} \Delta e_{i}^{2}}{m}} \\
& \operatorname{RMSE}_{u}=\sqrt{\frac{\sum_{i=1}^{m} \Delta u_{i}^{2}}{m}}
\end{aligned}
$$

where $m$ is the sample size, which is 30 and 42 for 24 and $4 \mathrm{~h}$ solutions, respectively.

\subsection{Long observation session ( 24 h-daily) results}

The purpose of this test is to evaluate contribution of GLONASS observations for the daily (24-h) data lengths. The data were analyzed from 12 January to 10 February 2015, which is 30 days in total. For each day a set of coordinates was determined based on both GPS-only and combined GPS/GLONASS observations for different elevation cut-off angles $\left(10^{\circ}\right.$, $20^{\circ}, 30^{\circ}$ and $40^{\circ}$ ). Since GNSS data used in this study were collected with a zero degree cut-off angle for all stations except LINZ (five degree cut-off angle), different elevation cut-off angles for all stations was set by the Bernese GNSS software. For this reason, elevation cut-off angle was the same for all stations during processes. In order to compare GPS-only and GPS/GLONASS scenarios the sampling rate of the raw input code and phase measurements was specified at $30 \mathrm{~s}$ with four different levels of elevation cut-off angle.

In order to examine the accuracy of the GPS-only and combined GPS/GLONASS solutions, RMSE values obtained in $(e, n, u)$ directions for the $24 \mathrm{~h}$ datasets are given in Fig. 3. As it can be seen from the Fig. 3 that GPS-only and GPS/GLONASS solutions produce similar results for the three components $(e, n, u)$. In case of $10^{\circ}, 20^{\circ}$ and $30^{\circ}$ elevation cut-off angle, RMSE values of the east and north components obtained from two network solutions are less than $5 \mathrm{~mm}$ for all stations, while RMSE of the height components are generally less than $20 \mathrm{~mm}$ for all stations, except WROC. In this case, it is also no significant difference in the RMSE value obtained from the GPS-only and GPS/ GLONASS solutions. In case of $40^{\circ}$ elevation cut-off angle, RMSE value for the three components is two to three times worse than the other cases. Note that GPS-only and GPS/ GLONASS solution is generally similar for the up component. In the east and north components, GPS-only solution produces similar results and even slightly better when compared to GPS/GLONASS solution (see LEIJ $(n)$ and LINZ $(e)$ ), while, GPS/GLONASS solution improve the RMSE for some stations, e.g. WROC $(n), \operatorname{LINZ}(e)$. It can be summarized from Fig. 3 that evaluation of the results reveals insignificant benefits of adding GLONASS observations in terms of accuracy for $24 \mathrm{~h}$ solutions.

\subsection{Short observation session $(4 \mathrm{~h})$ results}

In order to investigate the impact of data length on both positioning (GPS-only, GPS/ GLONASS) performance, the daily ( $24 \mathrm{~h}$ ) datasets, from 12 to 18 January 2015, were split into subsets of $4 \mathrm{~h}$ length $(0-4,4-8,8-12,12-16,16-20$ and 20-24 for 1 day). For each dataset a set of coordinates was determined based on both GPS-only and combined GPS/ GLONASS observations for different elevation cut-off angles $\left(10^{\circ}, 20^{\circ}, 30^{\circ}\right.$ and $\left.40^{\circ}\right)$. In this manner we processed 336 sessions, i.e., six four-hours subsets for a day times seven days times two solutions (GPS-only and GPS/GLONASS combined) times four different elevation cut-off angles.

Figure 4 shows the RMSE value obtained in East, North and Up directions for the $4 \mathrm{~h}$ datasets. It can be inferred from the Fig. 4 that GPS/GLONASS solution improves the 

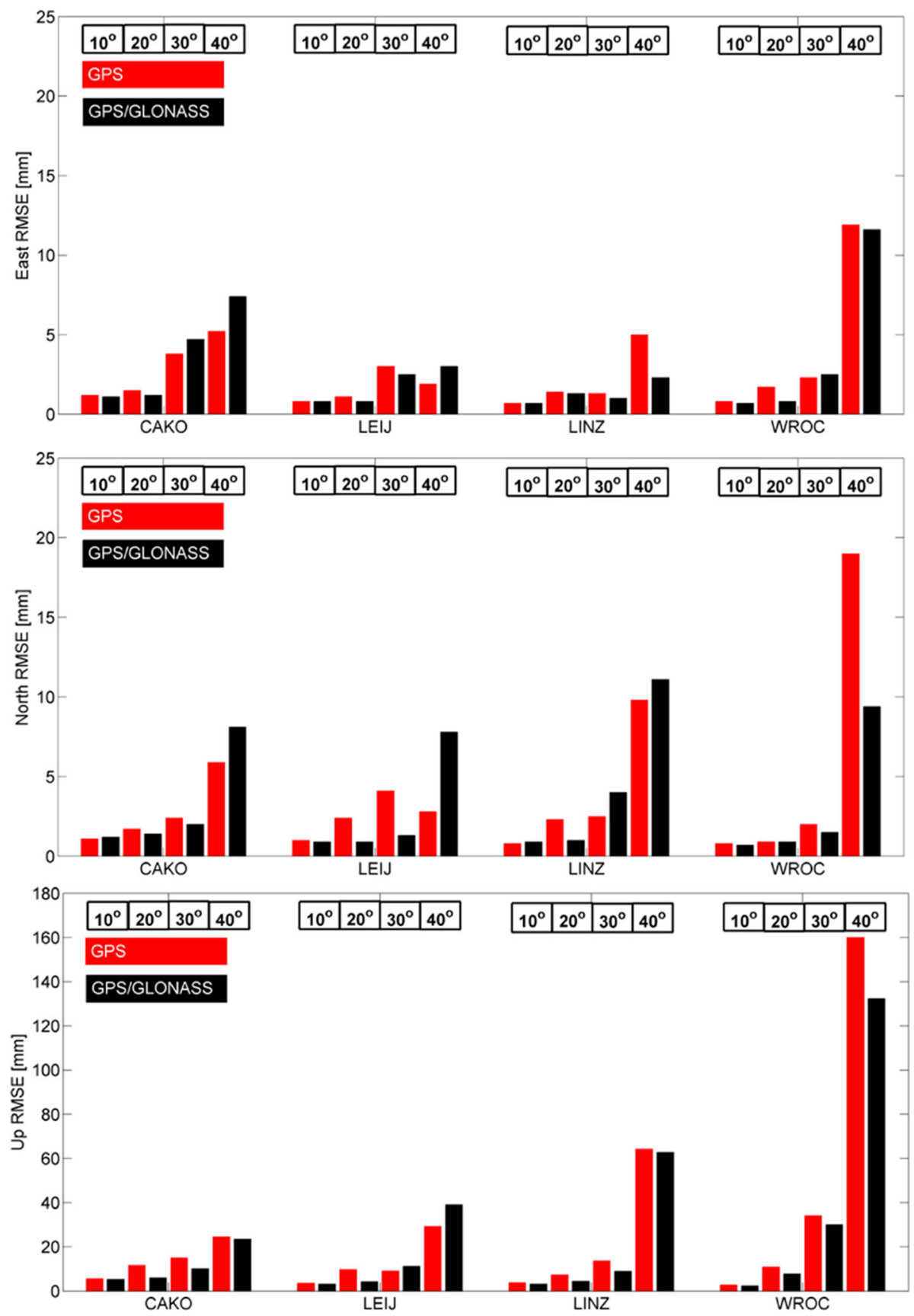

Fig. 3 RMSE values obtained in the East, North and Up directions for the $24 \mathrm{~h}$ datasets

RMSE, more or less, in the three (e, n, u) directions for all elevation cut-off angle cases. This indicates that the addition of GLONASS to GPS increases the accuracy of positioning in case of short observation session. RMSE values of the east and north components 

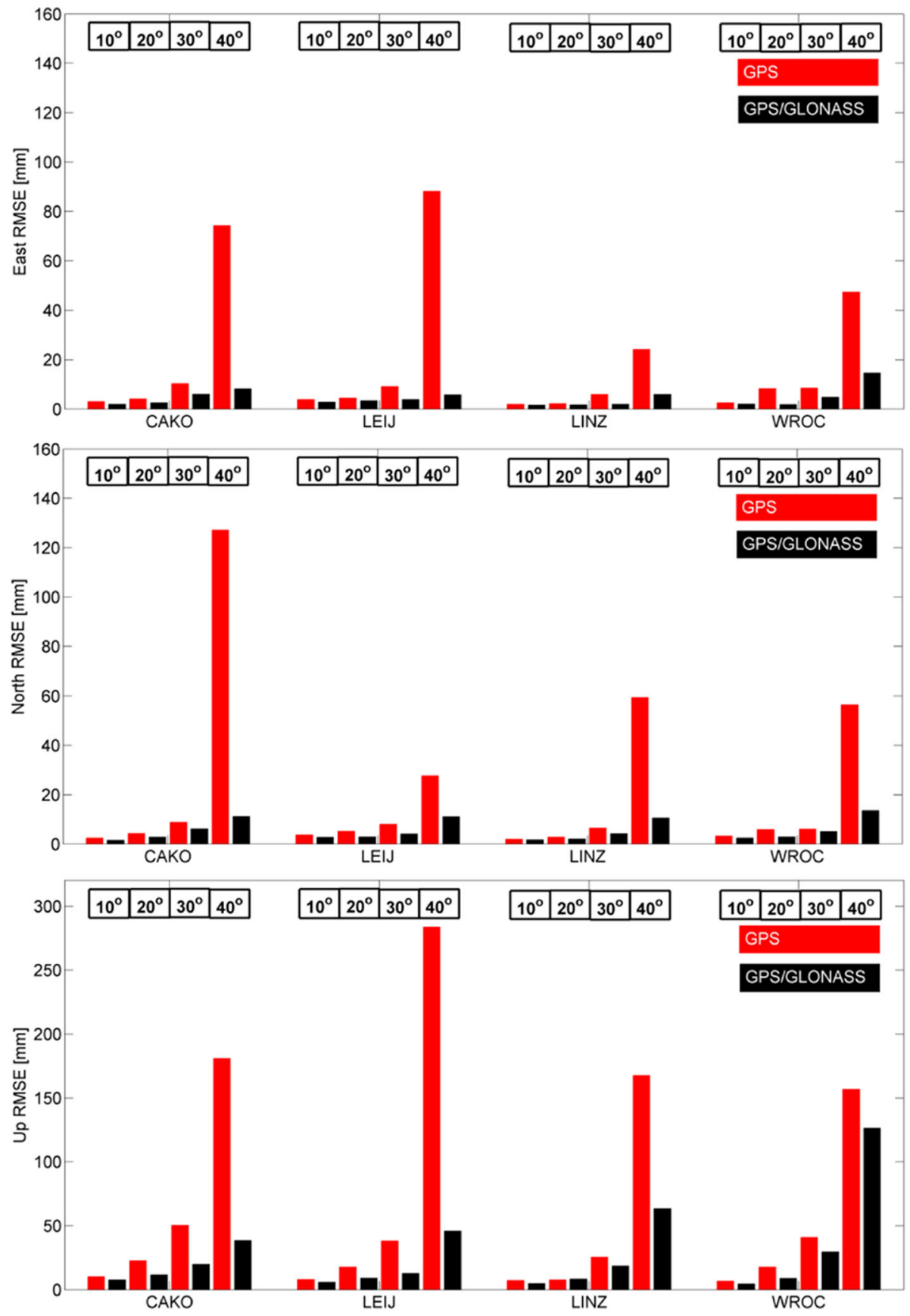

Fig. 4 RMSE values obtained in East, North and $U p$ directions for the $4 \mathrm{~h}$ datasets

obtained from both solutions are nearly similar in case of $10^{\circ}$ and $20^{\circ}$ elevation cut-off angle, while combined GPS/GLONASS solution is slightly better than GPS-only solution in case of $30^{\circ}$ elevation cut-off angle. For the up component, both solutions are almost 
similar in case of $10^{\circ}$ elevation cut-off angle, while combined GPS/GLONASS solution is slightly better for $20^{\circ}$ elevation cut-off angle and generally better for $30^{\circ}$ elevation cut-off angle. In case of $40^{\circ}$ elevation cut-off angle, note that the superiority of combined GPS/ GLONASS solution over GPS-only solution becomes more distinguished for the three $(e$, $n, u)$ components.

\subsection{Combined evaluation of daily and $4 \mathrm{~h}$ results}

In order to further analyze long and short term results, the number of satellites, PDOP, utilized observation numbers and RMSE corresponding to estimated coordinates are examined in this section.

In Fig. 5, the number of GPS, GLONASS and GPS/GLONASS combined satellites, with an elevation cut-off angle of $10^{\circ}, 20^{\circ}, 30^{\circ}$ and $40^{\circ}$, are given. As shown in Fig. 5, the number of visible satellites decreases when elevation cut-off angle increases. As it can be seen from Fig. 5, the number of visible GPS satellites is more than GLONASS satellites for most of the time. In case of the $10^{\circ}$ elevation cut-off angle, the number of observed satellites for GPS and GLONASS system varied from 6 to 12 and from 5 to 9, respectively. In case of the 40 degree elevation cut-off angle, the number of the observed satellites varied from 2 to 6 (GPS) and from 1 to 4 (GLONASS), respectively. In this case, the number of the satellites tracked by each system separately never exceeded 6 , sometimes even dropping to 1 . In case of higher elevation cut-off angles, the benefits of the combination of GLONASS with GPS will become more evident since less than four GPS satellites are available.

In Fig. 6, it can be seen that the PDOP (Positioning Dilution of Precision) for GPS-only changes substantially over this period of time when compared to PDOP for GPS/GLONASS. The combined satellite systems dramatically improve the PDOP value since the total number of satellite is increased and satellite geometry is getting better. Here, PDOP for GPS clearly shows data gaps in their time series due to the fact that the total number of visible satellites then drops below four. The PDOP of the combined system remains small.

Figure 7 shows the average number of observations used in the final processing step, without outlier observations. The average number of observation is calculated using all

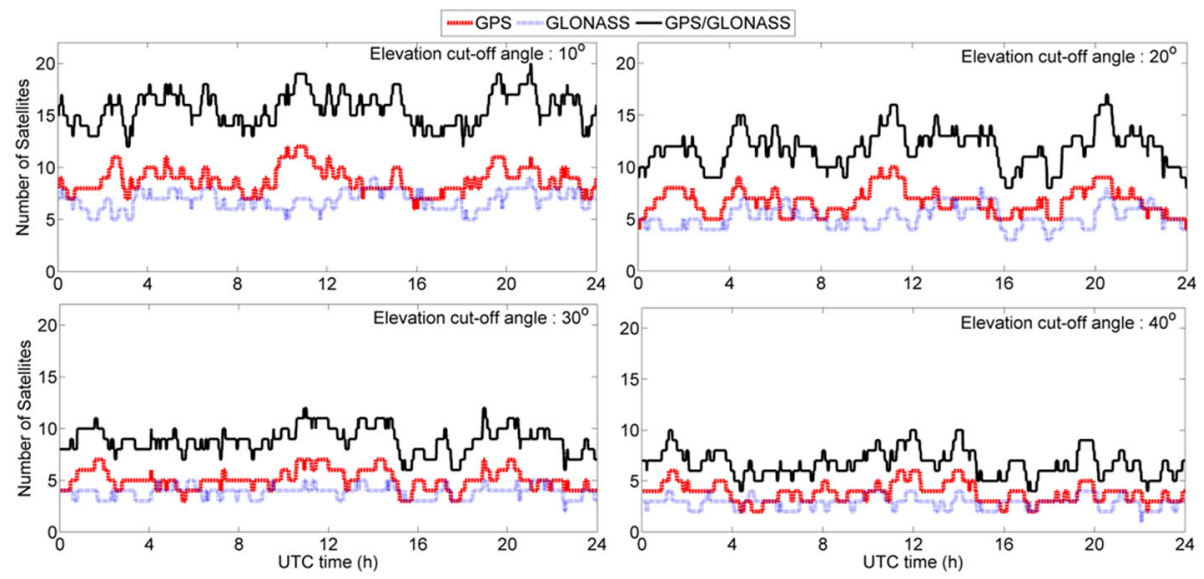

Fig. 5 Satellite visibility of GPS, GLONASS and GPS/GLONASS with $10^{\circ}, 20^{\circ}, 30^{\circ}$ and $40^{\circ}$ elevation cut-off angle for GOPE, January 12, 2015 


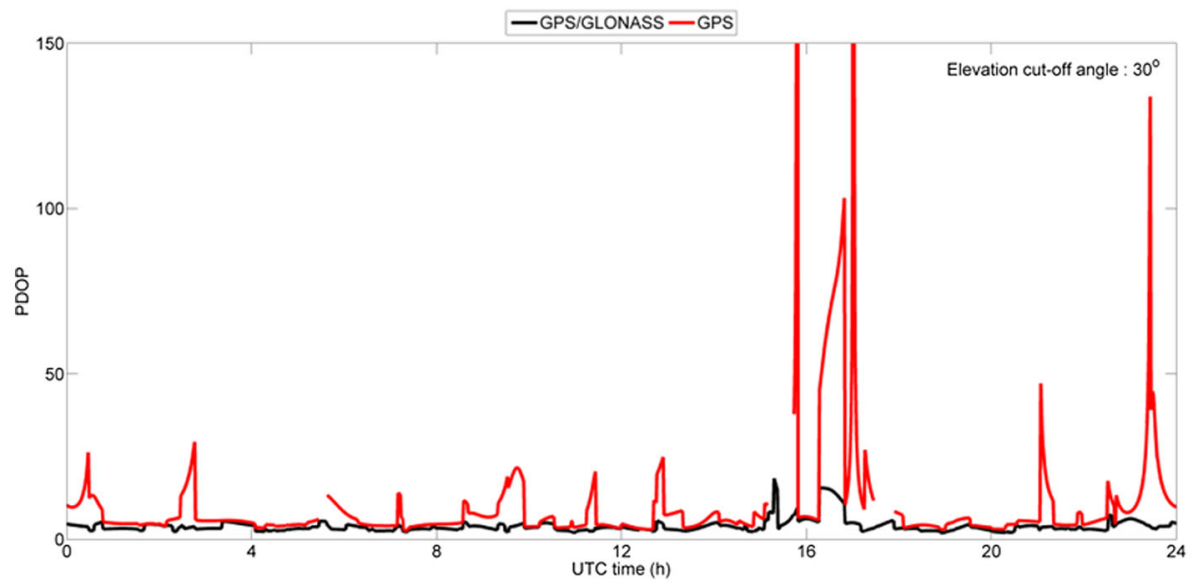

Fig. 6 PDOP for GPS and GPS/GLONASS with an elevation cut-off angle of $30^{\circ}$ for GOPE, January 12 , 2015

datasets. As depicted in Fig. 7, the number of observations in a combined GPS/GLONASS processing scenario for the daily observations is nearly two times higher than the GPS-only processing scenario for all sky view cases, whereas the number of observations in a combined GPS/GLONASS processing scenario for 4 hourly observations is nearly three times higher than the GPS-only processing scenario for $40^{\circ}$ elevation angle case (see $0-4$ and 20-24 time periods). The reason for this is that of the number of available GPS satellites is below the minimum requirement of four, in some moments for some time slices thus not being able to provide meaningful solutions and therefore they are eliminated. In addition, since the number of observations is one of the factors, directly influencing the RMSE of the estimated coordinates in Bernese GNSS software (version 5.2), the RMSE of the estimated coordinates based on final processing step of GPS-only data sets are at the

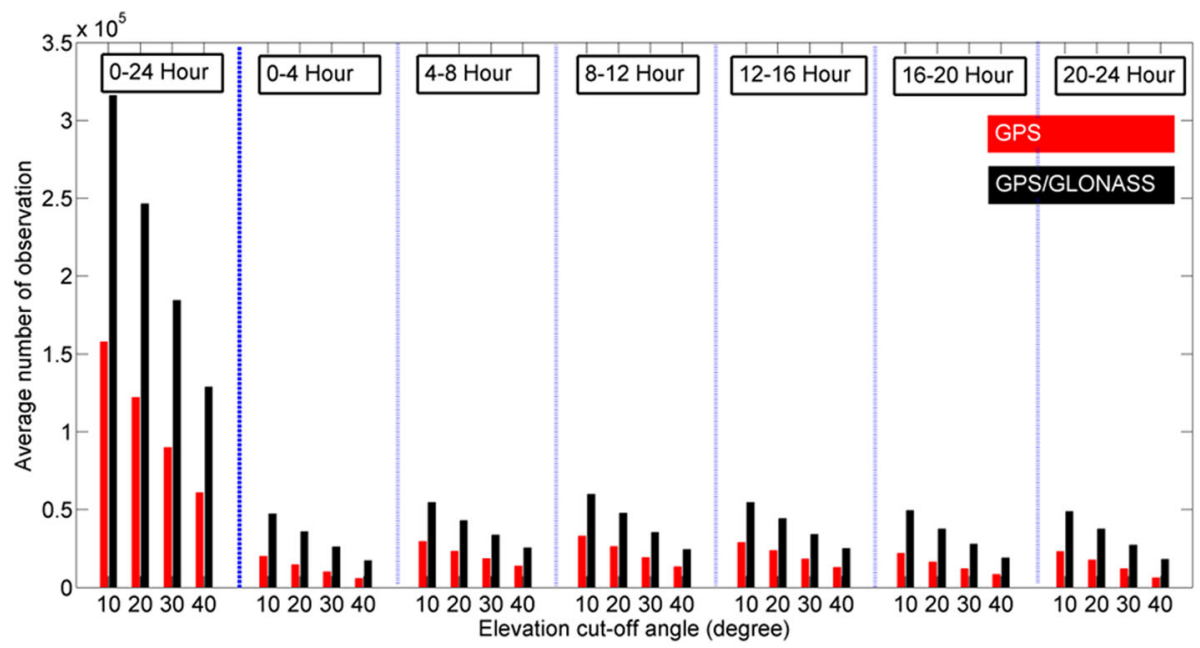

Fig. 7 Mean of the used observations according to the time slices 
larger scale particularly in case of the extremely limited conditions $\left(40^{\circ}\right)$. It can be inferred from 24 and $4 \mathrm{~h}$ applications, adding GLONASS observations has no clear impact on the accuracy of the estimated coordinates for $24 \mathrm{~h}$ solutions, whereas for limited and extremely limited sky view conditions based on $4 \mathrm{~h}$ processes, position and particularly height results are significantly improved in terms of accuracy by adding GLONASS observations. Thus it is worthwhile to apply combined data sets for short time slices and limited sky view conditions.

\section{Conclusion}

The performance of GPS-only yields reliable results under open sky view conditions. Therefore combined use of GPS/GLONASS systems for the positioning might be insignificant. However, the ability to utilize extra satellites available in the GLONASS system can enhance the performance of network-based positioning under various limited sky view conditions. In order to examine such conditions, this study attempts a comprehensive capability analysis of network-based positioning using GPS-only and combined GPS/GLONASS observations. Evaluation is based on results derived from the processing of long ( $24 \mathrm{~h}$, monthly) and short ( $4 \mathrm{~h}$, weekly) observation data sets with four different sky view conditions [open $\left(10^{\circ}\right)$, semi-open $\left(20^{\circ}\right)$, limited $\left(30^{\circ}\right)$ and extremely limited $\left(40^{\circ}\right)$ ]. Analysis based on $24 \mathrm{~h}$ observations exhibits similar results for GPS and GPS/GLONASS, in terms of accuracy for all sky view conditions. Generally speaking, the improvement of positioning accuracy, when adding GLONASS observation, in case of daily observation session is not apparent. However, for the shorter observation periods $(4 \mathrm{~h})$ the GPS/ GLONASS solutions suggest better accuracy indicators compared with their GPS-only equivalents particularly for the extremely limited sky view condition $\left(40^{\circ}\right)$. Finally, the results show that the combined GPS/GLONASS solutions in case of short observation session and limited sky view environment, such as urban canyons, mountain, forest and open-pit mines area, is required in order to obtain higher order of positioning and height accuracy, particularly for crustal and structural deformation monitoring applications.

Note that, in this research, all obstructed visibilities (e.g. $10^{\circ}, 20^{\circ}, 30^{\circ}$ and $40^{\circ}$ ) were generated from the data collected with zero and less than $5^{\circ}$ elevation cut-off angle, they may exhibit better positioning results than the data collected under real obstructed area conditions, such as urban canyon and deep open-pit mine. However, the results presented in this study confirm the contribution and advantages of GLONASS constellation to GPSonly in case of limited sky view condition and short observation session. Similar researches regarding network positioning can be carried out using other GNSS combination such as GPS/Galileo, GPS/BeiDou and can be compared with the results presented in this study.

Acknowledgments The authors are very grateful to anonymous reviewers for their constructive comments, which helped to improve the manuscript.

\section{References}

Alcay S, Inal C, Yigit CO, Yetkin M (2012) Comparing glonass-only with GPS-only and hybrid positioning in various length of baselines, acta Geod. Geop Hung 47:1-12. doi:10.1556/AGeod.47.2012.1.1

Alkan RM, Ilci V, Ozulu IM, Saka MH (2015) A comparative study for accuracy assessment of PPP technique using GPS and GLONASS in urban areas. Measurement 69:1-8 
Al-Shaery A, Lim S, Rizos C (2011) Assessment of network-based positioning performance using GPS alone versus GPS and GLONASS combined. In: Proceedings of the 24th international technical meeting of the satellite division of the institute of navigation (ION GNSS 2011), Portland, OR, September 2011, pp 2341-2349

Bruyninx C (2007) Comparing GPS-only with GPS + GLONASS positioning in a regional permanent GNSS network. GPS Solut 11:97-106

Choy S, Zhang S, Lahaye F, Héroux P (2013) A comparison between GPS-only and combined GPS + GLONASS precise point positioning. J Spatial Sci 58:169-190. doi:10.1080/14498596.2013. 808164

Dach R, Fridez P (2016) Bernese GNSS software version 5.2 Tutorial. Astronomical Institute, University of Bern, Bern

Dach R, Lutz S, Walser P, Fridez P (2015) Bernese GNSS software version 5.2, user manual. Astronomical Institute, University of Bern, Bern

Dawidowicz K, Krzan G (2014) Coordinate estimation accuracy of static precise point positioning using online PPP service, a case study. Acta Geod Geophys 49:37-55. doi:10.1007/s40328-013-0038-0

Leick A (1994) GPS Satellite Surveying, 2nd edn. Wiley, New York

Martin W, Ladd J (1997) GPS + GLONASS Surveying. Proceeding of ION GPS-97, Albuquerque, New Mexico

Raffaela C, Marco G (2000) Dual Frequency GPS + GLONASS Measurements in the static relative positioning. Boll geod e sci afini 59:391-403

Wang J, Wang J (2007) Comparing long baseline results from GPS and GPS/GLONASS. In: Combined International Symposium \& Exhibition on Geoinformation \& GNSS, Johor Bahru, 5-7 November CDROM procs, paper 59

URL-1 http://igs.org/products

Yigit CO, Gikas V, Alcay S, Ceylan A (2014) Performance evaluation of short to long term GPS/GLONASS and GPS/GLONASS post-processed PPP. Surv Rev 46:155-166. doi:10.1179/1752270613Y. 00000000068

Zheng Y, Nie G, Fang R, Yin Q, Yi W, Liu J (2012) Investigation of GLONASS performance in differential positioning. Earth Sci Inf 5:189-199. doi:10.1007/s12145-012-0108-9 